\title{
Tetralogy of Fallot: aorto-pulmonary collaterals and pulmonary arteries have distinctly different transcriptomes
}

\author{
Xiaoyuan Ma', Laura A. Barboza' ', Arpi Siyahian'1, Olaf Reinhartz'1, Katsuhide Maeda', Vadiyala Mohan Reddy' ${ }^{1}$ Frank L. Hanley ${ }^{1}$ \\ and Robert Kirk Riemer ${ }^{1}$
}

BACKGROUND: Tetralogy of Fallot patients with pulmonary atresia (TOF/PA) present a pulmonary blood supply directly from aortic collateral arteries. Major aorto-pulmonary collateral arteries (MAPCAs) present substantial clinical and surgical management challenges. Surgical operations to reestablish and promote further development of a pulmonary arterial connection preferentially utilize MAPCAs for reconstruction of central pulmonary arteries. However, the propensity of some MAPCAs to develop stenosis rather than growth may impair the response to reconstructions.

METHODS: Probe sets prepared from MAPCAs, PA, and aorta mRNA were used to interrogate human genome microarrays. We compared expression differences between pairs of the three vessels to determine whether MAPCAs display distinct expression patterns.

RESULTS: Functional clustering analysis identified differences in gene expression, which were further analyzed by gene ontology classification. A subset of highly regulated genes was validated using quantitative PCR. Expression differences among vessel types were observed for multiple gene classes. Of note, we observed that MAPCAs differentially express several genes at much higher levels than either PA or aorta.

CONCLUSION: MAPCAs differ from PA or aorta by significantly altered levels in gene expression, suggesting a transcriptional basis for their physiology that will guide a further understanding of the pathobiology of MAPCAs and TOF.

M ajor aorto-pulmonary collateral arteries (MAPCAs) are congenital systemic-to-pulmonary collateral arteries that supply the lung segments of patients with an abnormal pulmonary arterial tree, forming a unique but pathological bridge between the great vessels of the heart. MAPCAs are commonly present in tetralogy of Fallot (TOF) patients having both pulmonary atresia and ventricular septal defect (PA-VSD) $(1,2)$. Although their origin is controversial $(3,4)$, evidence supports the concept that MAPCAs are the remnants of intersegmental arteries supplying the lung buds in early fetal development, before the intraparenchymal arteries reach the central pulmonary arteries. It is thought that the intersegmental arteries normally regress when the central pulmonary to intraparenchymal artery connection is established. Because of the absence of the normal right ventricle to lung vasculature connection (i.e., presence of PA), the intersegmental arteries apparently continue in confluence with the intrapulmonary vasculature and persist beyond birth (5) as MAPCAs. The pattern of MAPCAs growth is observed to change after birth; their caliber tends not to increase over time as expected. Importantly, within the first few neonatal months they often become irregularly shaped, thickened, kinked, and stenotic, resulting in hypoperfused lung segments. As a consequence of the lack of enlargement of these collaterals, stenoses are usually present at the site of confluence with the pulmonary artery, somewhere between the aorta and the lung, or before their entrance into the parenchyma (6). These structural modifications may be triggered or accelerated by the postnatal decline in pulmonary vascular resistance, and subsequent increased blood flow velocity and shear stress. Myointimal hyperplasia is more likely to develop in the collaterals with extended exposure to systemic pressure and flow, leading to occlusion of the vessels and loss of lung segments if no other supply is available (7). Nonstenotic collaterals, on the other hand, supply the pulmonary vasculature under systemic pressure, which ultimately results in obstructive pulmonary vascular disease, loss of lung segments, and pulmonary hypertension (2,7-9).

Patients with VSD and MAPCAs present a total mixing lesion, which results in greater cardiac work due to volume overload, yet reduced systemic oxygen saturation, a condition that promotes a number of pathophysiologic consequences, including ventricular failure. Complex surgical operations are performed to reestablish the pulmonary flow connection, utilizing MAPCAs as raw material for reconstruction of the pulmonary arterial system. Currently, a unifocalization operation is a more-accepted management strategy for these patients. It consists of creating a one-compartment pulmonary arterial system, recruiting as many lung segments as possible by connecting the true pulmonary arteries and the MAPCAs system (10). The ultimate goal is to reestablish forward flow under pulmonary pressures to further the development of pulmonic arborization $(7,8,11,12)$. Before this approach, it was estimated that $65 \%$ of patients with PA-VSD and MAPCAs would survive to $1 \mathrm{y}$ of age and $50 \%$ to $2 \mathrm{y}$, regardless of surgical 
intervention (13). Poor technical reconstruction, including failure to address existing degenerated segments, and late timing are known reasons for a poor outcome after unifocalization (14). While collaterals left in their original site develop stenosis and obstructions, they tend to grow when unifocalized.

To date, no genome-wide expression profiles (transcriptomes) of vessel tissues from TOF patients have been reported. To better understand the pathophysiology of MAPCAs, we are investigating biologic differences between MAPCAs and their connecting vessels: aorta (AO) and pulmonary artery (PA). Here, we report a comparative analysis of gene expression in MAPCAs and their contiguous vascular connection, the pulmonary artery and aorta vessels. The analysis reveals multiple differences between MAPCAs and the great vessels of the heart.

\section{RESULTS}

\section{Comparison of Gene Expression Profiles}

We identified 193 differentially regulated genes in MAPCAs (fold $>2.0, P<0.05, t$-test, followed by Benjamini and Hochberg FDR correction) compared with the PA group, and 405 genes in the PA vs. aorta group comparison. However, the largest number of differentially regulated transcripts was observed in the MAPCAs vs. aorta group comparison, which had a total of 466 altered transcripts. The identities of the up- and downregulated genes and their fold-change cutoff are listed in Figure 1a. A comparison of the number of genes differentially regulated in the three vessel groups as well as genes common to the vessel types for two- and fourfold change thresholds are illustrated in Venn diagrams (Figure 1b,c). Only 20 genes were differentially expressed in common in all three groups (twofold change; Figure 1b); aorta had the highest number of regulated genes in common with either PA (192) or MAPCAs (111). At a fourfold change in expression however, there were no regulated genes common to all three tissues (Figure 1c). PA had 3 genes in common and aorta had 17 genes in common. Notably, MAPCAs had a higher number (33) of gene expression changes compared with either PA or aorta tissue, and the majority of genes are upregulated. Supplementary Table S1 online lists the genes expressed most highly in each tissue type and their comparative level in the other two tissues.

To investigate the potential relevance of the altered gene expression profiles of MAPCAs, we used gene ontology (GO) analysis to quantitate the representation of genes among three functional classifications: Molecular Function, Biological Process, and Cellular Components. Figure 2 illustrates that Biological Process was predominantly represented in the MAPCAs vs. PA group, while Cellular Components represented the largest class in both the MAPCAs vs. aorta and PA vs. aorta comparison groups.

Analysis using ExPlain 3.0 was employed to explore statistically overrepresented groups and identify significantly represented GO categories. In the MAPCAs vs. aorta and PA vs. aorta groups at a $P$ value cutoff of $<0.01$, approximately 200 GO classes were enriched in the upregulated gene set. Notably, the enriched GO classes include Vasculature Development, Angiogenesis, and Cell Proliferation. However at this cutoff level, only 1 GO class (Developmental Process) was enriched in both downregulated gene sets. In the MAPCAs vs. PA group as shown in Figure 3, a total of 22 GO classes were enriched in the upregulated gene set (138 genes), including Developmental Process, Extracellular Matrix, and Cell Differentiation, at the

\begin{tabular}{|ccccccc|}
\hline a & \multicolumn{2}{c}{ MAPCA/aorta } & \multicolumn{2}{c|}{ PA/aorta } \\
& Up change & Down & Up & Down & Up & Down \\
\hline$>2$ & 138 & 55 & 249 & 217 & 172 & 233 \\
$>4$ & 35 & 12 & 56 & 33 & 16 & 14 \\
$>10$ & 6 & 2 & 7 & 4 & 0 & 1 \\
\hline
\end{tabular}
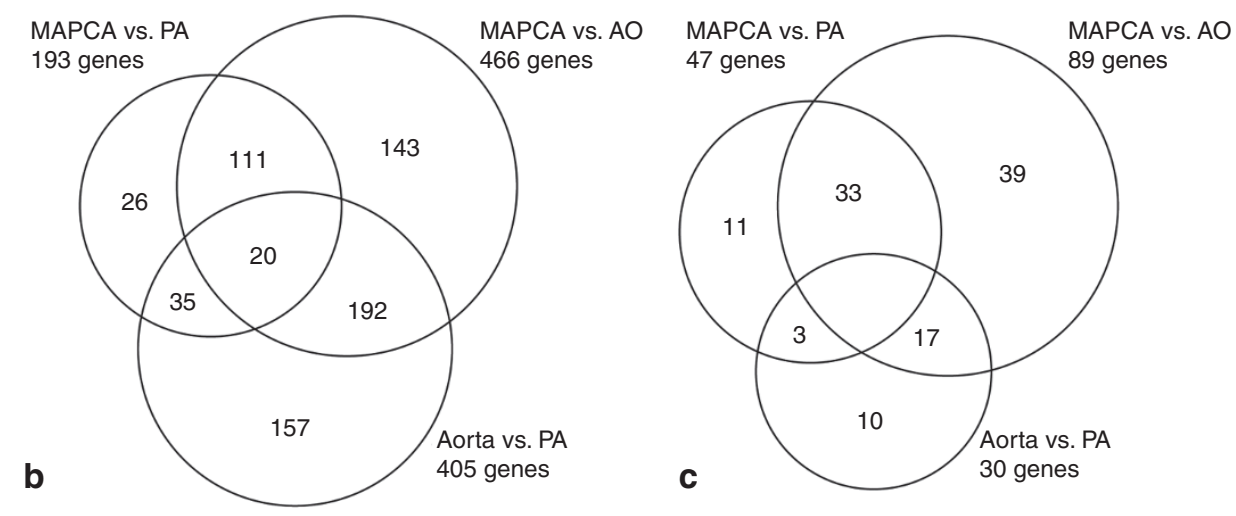

Figure 1. Integrated analysis of gene expression patterns. (a) Number of differentially regulated genes as a function of the fold-change cutoff value. Venn diagrams depict the genes with overlapping expression among three vessel types at a twofold change level (b), and a fold-change level (c) in the study group (one-way ANOVA corrected $P$ value $\leq 0.05$ ). AO, aorta; MAPCA, major aorto-pulmonary collateral artery; PA, pulmonary artery. 

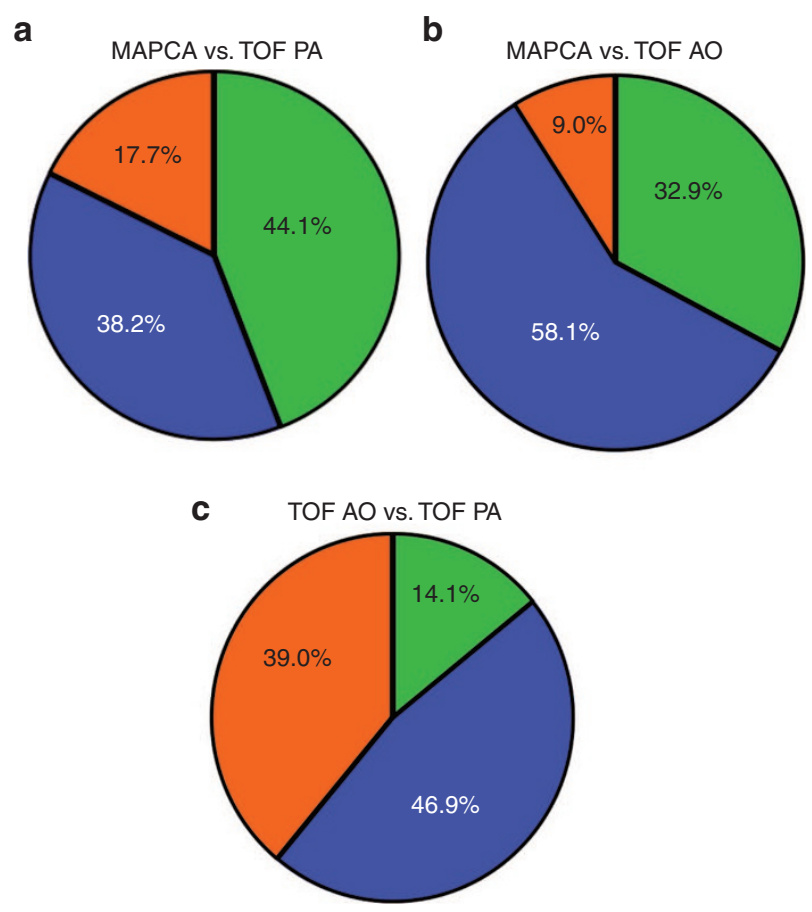

Figure 2. Functional classification of regulated genes. Representation of significant gene ontology $(\mathrm{GO})$ functional categories enriched for the differentially regulated genes in the three comparison groups: MAPCAs vs. TOF PA, MAPCAs vs. TOF aorta, and TOF aorta vs. TOF PA are shown. Orange shading represents Molecular Function, green represents Biological Process, and blue represents Cellular Component. AO, aorta; MAPCA, major aortopulmonary collateral artery; PA, pulmonary artery; TOF, tetralogy of Fallot.

$P$ value threshold of $<0.01$. Two GO classes were enriched (Developmental Process and Regulation of multicellular organismal process) in the downregulated gene set (55 genes). The most noteworthy GO classes in the highly regulated MAPCAs/PA pair (fold $>4.0, P<0.05$ ) gene set include BMP Signal Pathway, Transforming Growth Factor Regulation, and Extracellular Region.

\section{Overview of the Gene Expression Patterns}

To further examine whether MAPCAs gene expression segregated from these three anatomically contiguous vessels, hierarchical clustering analysis was performed based on the expression values to compare these three vessel groups. The expression heat map (Figure 4) shows the hierarchical clustering gene tree resulting from this analysis: the five lanes on the left are from MAPCAs samples, five samples from PA are in the middle lanes, and the three lanes on the right are from aorta samples. Of note, the clustering dendrogram at the top of Figure 4 shows that the PA and aorta groups are more closely related, even though the specimens are from different patients. Expression changes observed in the MAPCAs group were highly distinct from the other two groups despite the fact that PA and MAPCAs specimens are paired samples from the same patients, further indicating that MAPCAs have many unique changes in gene expression. Overall, the vessel types segregated into two major expression pattern groups indicated by the colored vertical bars at the right of the heat map, designated

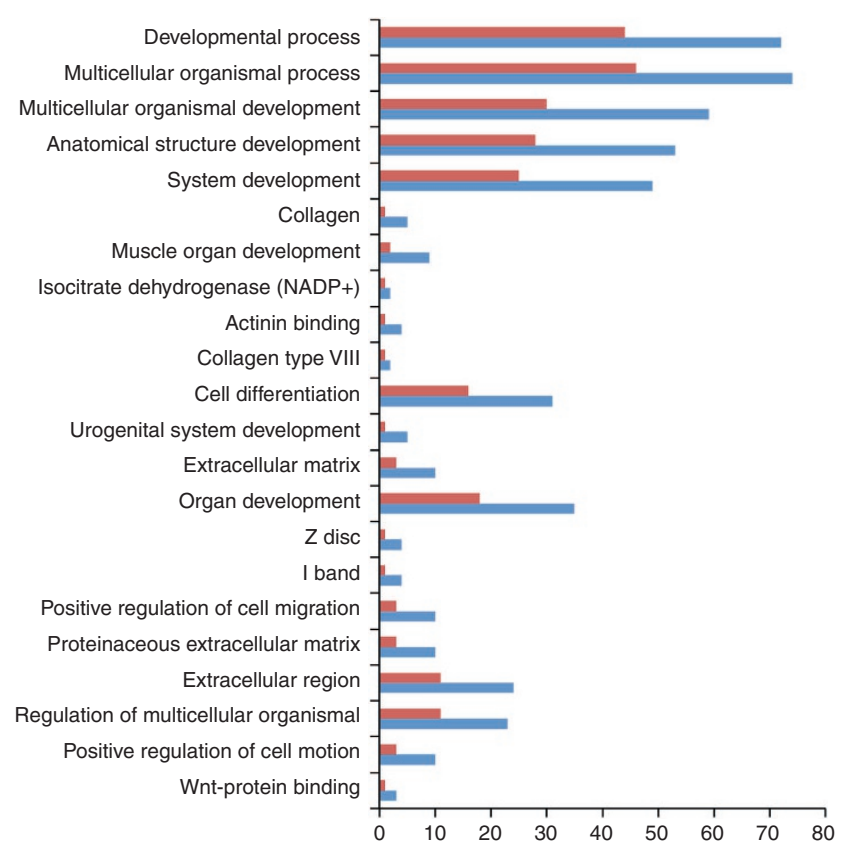

Figure 3. Gene ontology (GO) categories in MAPCAs/PA upregulated genes. Enriched GO categories in MAPCAs vs. PA group upregulated genes compared with expression of human housekeeping genes. Blue bars represent actual enriched number of genes, while red bars represent expected enrichment by chance. The $x$-axis indicates the number of genes in each category ("Hits").

a-d. Group 1 consists of two subsets: (a, red bar) MAPCAs and PA upregulated and aorta downregulated; and (d, yellow bar) MAPCAs and PA downregulated and aorta upregulated. Group 2 (blue bars), which we designated as the MAPCAsspecific gene pattern, consists of two subsets: (b) MAPCAs upregulated, PA and aorta downregulated and (c) MAPCAs downregulated but PA and aorta upregulated.

\section{Functional Gene Pathway Analysis Identifies Key Canonical Pathways Altered in MAPCAs}

We have conducted functional analysis of genes with over twofold altered expression in the three vessel sample groups using Ingenuity Pathway Analysis software as well as the BioBase biological database and have identified several distinct relationships. The major gene network functions identified in both the MAPCAs vs. PA and MAPCAs vs. aorta comparison groups were Cardiovascular system development and function, Organismal development, and Tissue development. Cancer, Cell death and survival, and Nervous system development and function were the major groups represented in the PA vs. aorta comparison group. The statistically significant canonical pathways in each of the three tissue types are shown in Supplementary Tables S2-S4 online. Interestingly, we observed no statistically significant canonical pathway in the group of genes with over fourfold altered expression.

\section{Confirmation of MAPCAs-Regulated Genes by RT-PCR}

To confirm the differential expression observed in microarray experiments, we performed QRT-PCR analysis of eight 


\section{Articles $\mid$ Ma et al.}

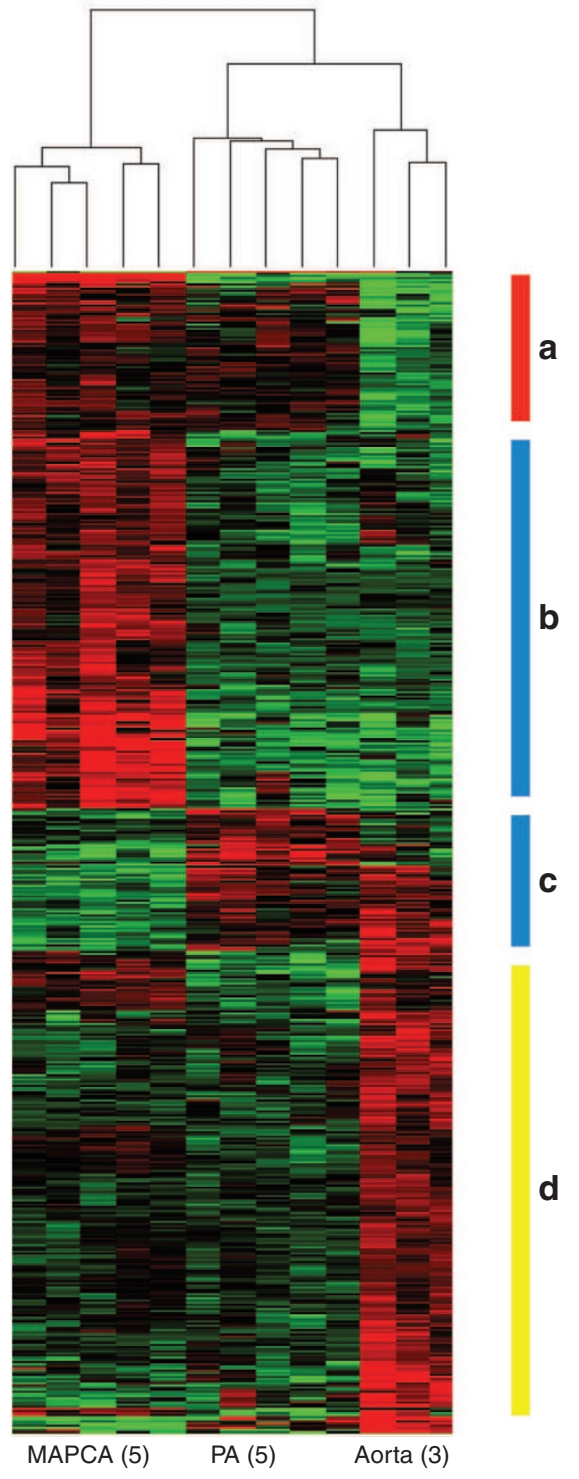

Figure 4. Heat map with clustering based on all detectable genes in tetralogy of Fallot (TOF) vessel tissue. Heat map (red, higher expression; green, lower expression) with hierarchical clustering based on fold $\geq 2.0$, $P \leq 0.05$ genes in the TOF specimens. The dendrogram at the top of the figure indicates relative group similarity. Stratification of vessel-specific expression patterns into two major groups is indicated by the colored vertical bars (a-d) at the right of the heat map. Group 1 consists of two subsets: red bar (a) MAPCAs and PA upregulated, and aorta downregulated; and yellow bar (d) MAPCAs and PA downregulated, and aorta upregulated. Group 2 (blue bars), designated as MAPCAs-specific gene type: (b) MAPCAs upregulated, and PA and aorta downregulated; and (c) MAPCAs downregulated, and PA and aorta upregulated.

representative genes: five upregulated genes, i.e., four and a half LIM domain-5 (FHL5), calsequestrin-2 (CASQ2), WAP four-disulfide core domain-1 (WFDC1), synaptotagminlike-2 (SYTL2), and calcium binding protein-1 (CABP1), and three downregulated genes, i.e., gremlin-1 and -2 (GREM1, GREM2) and secreted frizzled-related protein-1 (sFRP1). The upregulation was confirmed in all five genes by real-time quantitative RT-PCR. Two of three downregulated genes show highly concordant quantitative measurements (Figure 5). For

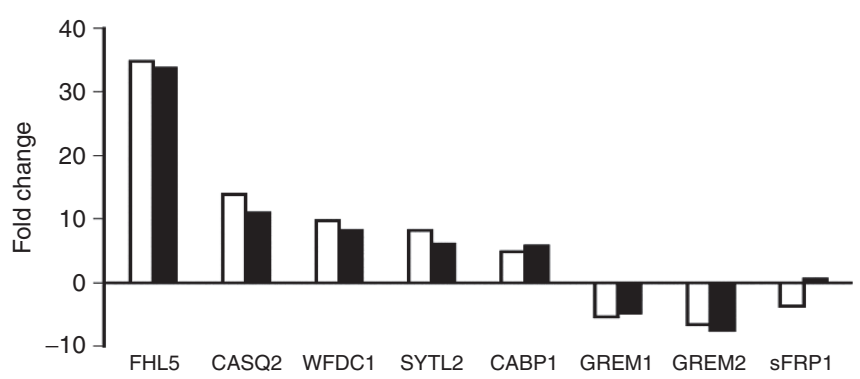

Figure 5. Validation of microarray data by qPCR. Data representing the relative fold expression changes of eight selected genes determined by real-time RT-PCR in mRNA prepared from five PA and five MAPCAs specimens after normalization to 18s RNA expression. Data are presented as mean fold expression in MAPCAs compared with PA. Open bars indicate fold change assessed by microarray. Filled bars indicate fold change determined by PCR.

the eight confirmed genes, these QRT-PCR results represent a $90 \%$ concordance with the microarray data (i.e., highest expression level of those genes with high signal intensity on the microarray).

\section{DISCUSSION}

This study was conducted to identify the differentially expressed genes among three contiguous vessel types in tetralogy of Fallot patients with pulmonary atresia (TOF/PA) in order to determine whether there are distinct genetic differences among these arteries. The major focus was on MAPCAs because they are vessels unique to TOF disease. Two general features were evident from transcriptome comparisons. First, the majority of gene expression ratios were higher $(>70 \%)$ in the MAPCAs vs. PA group, suggesting a general upregulation in gene expression in MAPCAs. Second, a much greater number of regulated genes were present in the aorta group compared with either PA or MAPCAs, and the proportion of upregulated or downregulated genes was similar in both comparison groups. A large number of highly regulated genes, with the majority upregulated in MAPCAs, were noted in this study.

The top GO functional classification showed an enrichment of genes involved in the BMP signal pathway including Grem1, Grem2, and Aldh1a2. IL13ra2 and Spint2 genes are associated with transforming growth factor regulation and the extracellular region. The marked downregulation of the BMP antagonist proteins Grem 1 and 2 is suggestive of the loss of a normally negative regulation of BMP signaling by preventing their interaction with their cell surface receptors. However, gremlin has also been shown to be capable of pro-angiogenic activity through BMP-independent mechanisms (15).

We found that MAPCAs express several genes at levels significantly greater than in either PA or aorta, demonstrating that MAPCAs may indeed be distinguished from up- and downstream contiguous vessels. The genes observed to be most highly regulated have been shown to play a role in vascular biology and disease. FHL5 is also known as ACT, Activator of CREM (cAMP response element modulator) in murine Testes, and is reportedly involved in the induction of vascular smooth muscle cell growth and intimal hyperplasia in human 
Table 1 Real-time PCR assays-on-demand reagent identifications

\begin{tabular}{llc}
\hline Gene & Assay ID (human) & Amplicon length (bp) \\
\hline FHL5 & Hs00220910_m1 & 97 \\
CASQ2 & Hs00415779_m1 & 96 \\
SYTL2 & Hs00909223_m1 & 107 \\
CABP1 & Hs00258552_m1 & 68 \\
WFDC1 & Hs00221849_m1 & 92 \\
sFRP1 & Hs00610060_m1 & 130 \\
GREM1 & Hs00171951_m1 & 80 \\
GREM2 & Hs01934663_s1 & 108 \\
\hline
\end{tabular}

vein graft samples (16). CASQ2 is a $\mathrm{Ca}^{2+}$-storage/buffer protein known to be present in the sarcoplasmic reticulum of cardiac muscle cells. CASQ2 controls the influx and efflux of $\mathrm{Ca}^{2+}$ from the sarcoplasmic reticulum in maintaining the intracellular and sarcoluminal $\mathrm{Ca}^{2+}$ concentration (17). We speculate that upregulation of CASQ2 in MAPCAs may promote their dilation.

In this report of our initial investigations of the transcriptional profile of MAPCAs and the vessels to which they commonly connect, we report distinct transcriptome differences, many of which we believe will be worthy of further investigation. However, we acknowledge that the study has a limitation in that other potentially relevant vessel types from patients with and without TOF were necessarily not available for comparison. It is our intention to continue to broaden this study as tissue and resources allow, so that a fuller understanding of collaterals from various systemic origins, as well as other, apparently normal vessels may be achieved. Further, the biological significance of the highly overexpressed nature of the specific MAPCAs genes we report here still remains to be explored. In most cases, matched tissue specimens from the same patient could not be obtained for analysis, as their availability is not predictable. Therefore the influence of interpatient variation on the data cannot yet be assessed. For the five matched pairs we analyzed by gene-specific PCR, the data were similar to those of unpaired samples. Although pairing of tissue samples potentially provides valuable additional insight and may reduce signal "noise," it also necessarily induces a degree of bias compared with randomized sampling.

In summary, our study compared gene expression differences among these three contiguous vessel types in order to determine if there are genetic differences among these arteries. We determined that MAPCAs express several genes at levels significantly greater than in either PA or aorta, demonstrating that MAPCAs may indeed be distinguished from up- and downstream contiguous vessels. Furthermore, the GO analysis revealed a complex pattern of gene expression and signaling pathways involved in MAPCAs biology.

The results presented in this study demonstrate, for the first time, specific genetic differences in MAPCAs biology that may ultimately help to explain their aberrant growth response to flow. As the first report focusing on the genes expressed these unusual vessels, it is an important initial step to understanding the complex biology of MAPCAs. We believe that a characterization of MAPCAs at a tissue and cellular level may enable the identification of key factors in their unusual negative response to blood flow, a characteristic that is unpredictable and presents challenges to repairs utilizing them as autograft materials.

\section{METHODS}

\section{Patient Characteristics}

Pulmonary arteries, collaterals, and aortic tissue samples were collected from patients undergoing surgical repair operations for TOF/ PA, MAPCAs. Specimens removed during surgical operations were collected under a protocol approved by the Internal Review Board for Human Subjects of Stanford University Medical Center, transported from the operating suite to the laboratory in cold saline immersed in ice, and portions were immediately stored at $-80^{\circ} \mathrm{C}$ for later mRNA analysis. Our patients were children less than 2 y of age with tetralogy of Fallot requiring surgical reconstruction. A total of 21 patients (35 specimens) with TOF/PA, MAPCAs (mean age $229 \mathrm{~d}$, range 24-547 days) undergoing primary corrective RVOT reconstruction for tetralogy of Fallot and unifocalization of pulmonary arteries and MAPCAs were studied by microarray and/or PCR validation. There were no significant differences in age, sex, and surgical procedure between PA, MAPCAs, and aorta groups. Where possible, paired PA and MAPCAs samples obtained from the same patient were used for microarray study to minimize possible individual- or disease-specific confounding factors. Aortic samples were generally not paired with PA in patients and rarely paired with MAPCAs; i.e., we used aortic tissue from different patients for microarray study.

\section{Analysis of mRNA Expression Profiles Using Microarrays}

Five paired PA and MAPCAs specimens and three individual aorta specimens were used to characterize vessel-specific transcriptomes (i.e., sets of expressed genes defined by genome-wide microarray analyses of total RNA). Total RNA was isolated with TRIzol reagent (Invitrogen) according to the manufacturer's protocols, followed by an additional RNA purification with an RNAeasy Mini Kit (Qiagen, Valencia, CA). The quality of RNA was assessed by UV absorption spectrophotometry (NanoDrop Technologies, ThermoFisher) and on-chip electrophoretic separation and quantitation (Bioanalyzer 2100, Agilent Technologies, Santa Clara, CA). Only samples having an A260/A280 ratio between 1.8 and 2.1 and a 28S/18S ratio within 1.5-2 were further processed at Stanford University Medical School Protein and Nucleic Acid Research core facility using standard protocols for cRNA labeling, hybridization, and detection procedures to probe Affymetrix GeneChip Human Genome U133 Plus 2 cDNA array slides (Affymetrix, Santa Clara, CA). The GeneSpring software suite (Agilent Technologies) was used for statistical analysis and normalization of microarray data. In brief, raw array data CEL files were uploaded to the GeneSpring GX version 11.0.2. Probelevel data were then compiled, normalized, and transformed using RMA. The following criteria were applied to filter the differentially expressed transcript list: a fold change of $>1.5$ and a Wilcoxon rank sum test where $P<0.05$ with Benjamini-Hochberg estimation of false discovery rate. The resulting list of differentially expressed transcripts was uploaded into the BioBase ExPlain 3.0 data system (BIOBASE GmbH, Germany) and Ingenuity IPA (Ingenuity Systems, Redwood City, CA) as the starting points for gene ontogeny analysis and generation of biological networks. In both systems, a $P$ value is calculated to estimate the probability that each biological function and/or disease assigned to the dataset of interest is not due to chance alone. Selected genes were visualized in a molecular network using information contained in IPA and show the connectivity of the individual proteins. Additionally, differentially expressed transcripts and genes are classified either by proprietary ontologies or by MESH terms according to the categories of canonical pathways, therapeutic target, biomarker, and molecular mechanism. The statistical significance of the association between the dataset and the categories is estimated from the ratio of the number of proteins from the dataset that map to the category divided by the total number of 
proteins that map to the canonical pathway. To define and identify patterns of gene expression profiles among all three vessel groups, unsupervised hierarchical clustering analysis was performed based on the value of the differentially expressed genes to determine the relationship of the samples.

\section{Real-Time PCR Quantitation of mRNA}

Total RNA prepared as described above was reverse transcribed (RT) to cDNA using the Superscript II RNase H Reverse Transcriptase kit (Invitrogen, Carlsbad, CA). Real-time PCR was performed in triplicate. An initial RT reaction minus RNA template confirmed that all RNA samples were free of contaminating genomic DNA. The Eukaryotic 18S rRNA (VIC/TAMRA) reagent kit was used as the endogenous control gene according to the TaqMan PCR protocol (Applied Biosystems, Foster City, CA). Probes and primers (Table 1) were obtained from ABI (Assay-on-Demand Gene Expression Assays). Reactions were run on an ABI 7900 Sequence Detection System and mRNA levels were determined using the standard curve method (ABI Prism 7900 Sequence Detection System).

\section{SUPPLEMENTARY MATERIAL}

Supplementary material is linked to the online version of the paper at http:// www.nature.com/pr

\section{STATEMENT OF FINANCIAL SUPPORT}

This work was supported in part by a grant (to A.S.) from the Lucile Packard Children's Hospital Heart Center Research Fund.

Disclosure: The authors declare no conflict of interest.

\section{REFERENCES}

1. Reinhartz O, Reddy VM, Petrossian E, et al. Unifocalization of major aortopulmonary collaterals in single-ventricle patients. Ann Thorac Surg 2006;82:934-8; discussion 938-9.

2. Tchervenkov CI, Salasidis G, Cecere R, et al. One-stage midline unifocalization and complete repair in infancy versus multiple-stage unifocalization followed by repair for complex heart disease with major aortopulmonary collaterals. J Thorac Cardiovasc Surg 1997;114:727-35; discussion 735-7.

3. Boshoff D, Gewillig M. A review of the options for treatment of major aortopulmonary collateral arteries in the setting of tetralogy of Fallot with pulmonary atresia. Cardiol Young 2006;16:212-20.

4. Nørgaard MA, Alphonso N, Cochrane AD, Menahem S, Brizard CP, d'Udekem Y. Major aorto-pulmonary collateral arteries of patients with pulmonary atresia and ventricular septal defect are dilated bronchial arteries. Eur J Cardiothorac Surg 2006;29:653-8.

5. Thiene G, Frescura C, Bortolotti U, Del Maschio A, Valente M. The systemic pulmonary circulation in pulmonary atresia with ventricular septal defect: concept of reciprocal development of the fourth and sixth aortic arches. Am Heart J 1981;101:339-44.

6. Haworth SG. Collateral arteries in pulmonary atresia with ventricular septal defect. A precarious blood supply. Br Heart J 1980;44:5-13.

7. Reddy VM, Liddicoat JR, Hanley FL. Midline one-stage complete unifocalization and repair of pulmonary atresia with ventricular septal defect and major aortopulmonary collaterals. J Thorac Cardiovasc Surg 1995;109:832-44; discussion 844-5.

8. Carotti A, Di Donato RM, Squitieri C, Guccione P, Catena G. Total repair of pulmonary atresia with ventricular septal defect and major aortopulmonary collaterals: an integrated approach. J Thorac Cardiovasc Surg 1998;116:914-23.

9. Macartney F, Deverall P, Scott O. Haemodynamic characteristics of systemic arterial blood supply to the lungs. Br Heart J 1973;35:28-37.

10. Malhotra SP, Hanley FL. Surgical management of pulmonary atresia with ventricular septal defect and major aortopulmonary collaterals: a protocolbased approach. Semin Thorac Cardiovasc Surg Pediatr Card Surg Annu 2009:145-51.

11. Moritz A, Marx M, Wollenek G, Domanig E, Wolner E. Complete repair of PA-VSD with diminutive or discontinuous pulmonary arteries by transverse thoracosternotomy. Ann Thorac Surg 1996;61:646-50.

12. Reddy VM, Petrossian E, McElhinney DB, Moore P, Teitel DF, Hanley FL. One-stage complete unifocalization in infants: when should the ventricular septal defect be closed? J Thorac Cardiovasc Surg 1997;113:858-66; discussion 866-8.

13. Bull K, Somerville J, Ty E, Spiegelhalter D. Presentation and attrition in complex pulmonary atresia. J Am Coll Cardiol 1995;25:491-9.

14. Hanley FL. MAPCAs, bronchials, monkeys, and men. Eur J Cardiothorac Surg 2006;29:643-4.

15. David L, Feige JJ, Bailly S. Emerging role of bone morphogenetic proteins in angiogenesis. Cytokine Growth Factor Rev 2009;20:203-12.

16. Nakanishi K, Saito Y, Azuma N, Sasajima T. Cyclic adenosine monophosphate response-element binding protein activation by mitogen-activated protein kinase-activated protein kinase 3 and four-and-a-half LIM domains 5 plays a key role for vein graft intimal hyperplasia. J Vasc Surg 2013;57:182-93, 193.e1-10.

17. Faggioni M, Knollmann BC. Calsequestrin 2 and arrhythmias. Am J Physiol Heart Circ Physiol 2012;302:H1250-60. 